\title{
非晶态合金穆斯堡尔谱的非对 称性及改进的拟合方法
}

\author{
徐祖雄马如璋 \\ (北京钢铁学院) \\ 李 士 \\ （中国科学院高能物理研究所，北京）
}

目前,在提高铁磁性非晶合金的 ${ }^{37} \mathrm{Fe}$ 穆斯堡尔谱的拟合优度方面, 尚存在两个有待进一步 解决的问题: 谱的六个宽峰的高度和线宽相对于谱中心的非对称性; 用 Hesse 法 ${ }^{[1]}$ 或 Window 法 $^{[2]}$ 拟合时, 如何合理地修正基本亚谱的峰面积比、峰宽、平均同质异能移位等参数. 本文分 析了非晶合金谱的非对称性,并提出了提高拟合质量的改进拟谱方法.

\section{一、非晶合金谱的非对称性}

大多数铁磁性非晶合金的 ${ }^{57} \mathrm{Fe}$ 穆斯堡尔谱都有一定程度的非对称性. 按六个宽峰的峰宽 $\Gamma_{K}(K-1,2, \cdots 6)$, 一般将谱的非对称性分为两大类: $\Gamma_{1}>\Gamma_{6}, \Gamma_{2}>\Gamma_{5}, \Gamma_{3}>\Gamma_{4}$, 例如 $\left(\mathrm{Fe}_{0.2} \mathrm{Co}_{0.8}\right)_{80} \mathrm{P}_{13} \mathrm{Al}_{3}^{[3]}$ 和 $\mathrm{Fe}-\mathrm{P}^{(4)}$ 非晶合金; $\Gamma_{1}>\Gamma_{6}, \Gamma_{2}<\Gamma_{5}, \Gamma_{3}<\Gamma_{4}$, 例如 $\mathrm{Fe}_{80} \mathrm{~B}_{20}{ }^{[5]}$ 非晶合 金. 已有部分文献提出用超精细场 $H_{\mathrm{hf}}$ 与同质异能移位 $\delta$ 的线性关系 ${ }^{[3.4]}$, 超精细场 $H_{\mathrm{hf}}$ 与四 极辟裂 $\triangle E Q$ 的线性相关 ${ }^{[5]}$ 或用偶极子场 $H_{a}{ }^{[6]}$ 解释上述两种非对称性.

我们的拟谱实践表明，仅用上述假设中的一种不足以满意地解释大多数非晶态合金谱的 非对称性. 三个超精细参数 $H_{\mathrm{h} f} 、 \triangle E Q$ 和 $\delta$ 均取决于铁原子的近邻组态,三者的分布之间必 然同时存在密切的相关性. 因此, 假设三个超精细参数 $H_{\mathrm{hf}} 、 \Delta E Q$ 和 $\delta$ 之间同时存在着下述 近似的线性关系更为合理:

$$
\Delta E Q=a \cdot H_{\mathrm{hf}}+c, \delta=b \cdot H_{\mathrm{hf}}+d .
$$

如果假设电场梯度轴向不对称参数 $\eta=0$, 则相应于某个超精细场 $H_{\mathrm{hf}}$ 的亚谱的每个次峰 蜂位为

$$
P_{K}-H_{\mathrm{hf}} \cdot\left(\alpha_{K}+a \cdot \beta_{K}+b\right)+\left(c \cdot \beta_{K}+d\right), \quad(K=1,2, \cdots, 6),
$$

式中的 $\alpha_{K}$ 和 $\beta_{K}$ 分别为超精细场 $H_{\mathrm{hE}}$ 和四极辟裂 $\Delta E Q$ 对峰位的影响系数. 由式 (2) 可 知, 常数 $c$ 和 $d$ 仅影响六个宽峰的峰位而与谱的非对称性无关. 常数 $a$ 和 $b$ 称之为谱的非对 称性参数,它们的大小和正负决定着谱线非对称性的类型和大小。即使对于 $\mathrm{Fe}_{8} \mathrm{~B}_{20}$ 和 $\mathrm{Fe}_{8} \mathrm{P}_{13}$ 这两种典型非晶合金, 也只有同时考虑 $H_{\mathrm{hf}}-\delta$ 和 $H_{\mathrm{hf}}-\Delta E Q$ 两种线性关系才能获得好的 拟合质量(图 1); 否则, 对 $\mathrm{Fe}_{80} \mathrm{~B}_{20}$ 仅按 $H_{\mathrm{hf}}-\triangle E Q$ 线性关系和对 $\mathrm{Fe}_{82} \mathrm{P}_{18}$ 仅按 $H_{\mathrm{hf}}-\delta$ 线性 关系拟合时, 如果使谱的第一、六宽峰拟合良好,则理论谱的第四、五宽峰的高度将高于实验谱

本文 1984 年6 月6 日收到. 
的,而第二、三宽峰的高度将低于实验谱的. 拟谱实践表明,通过选择合适的 $a$ 和 $b$ 值, 可以有 故地提高各种非晶合金非对称性谱的拟合质量. 表 1 列出了几种常见非晶合金谱拟合最佳时 的参数 $a$ 和 $b$.

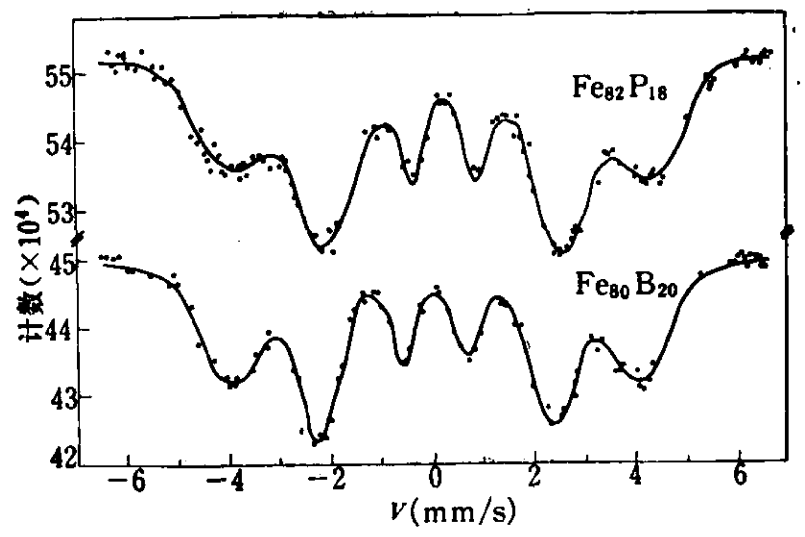

图 $1 \mathrm{Fe}_{22} \mathrm{P}_{10}$ 和 $\mathrm{Fe}_{20} \mathrm{~B}_{20}$ 的非对称性谱

表 1 几种非晶合金谱的非对称性参数 $a$ 和 $b$

\begin{tabular}{|c|c|c|c|c|c|}
\hline 合 金 & $\mathrm{Fe}_{40} \mathrm{Ni}_{40} \mathrm{P}_{14} \mathrm{~B}_{6}$ & $\mathrm{Fe}_{82} \mathrm{P}_{10}$ & $\mathrm{Fe}_{80} \mathrm{~B}_{20}$ & $\mathrm{Fe}_{78} \mathrm{Si}_{10} \mathrm{~B}_{12}$ & $\mathrm{Fe}_{81} \mathrm{~B}_{13,5} \mathrm{Si}_{3,9} \mathrm{C}_{2}$ \\
\hline$a\left(\times 10^{-3}\right)$ & -0.86 & -1.0 & -1.0 & -1.1 & -0.9 \\
\hline$b\left(\times 10^{-3}\right)$ & -0.43 & -0.6 & 0.4 & 0.5 & 0.5 \\
\hline
\end{tabular}

\section{二、改进的拟合程斯堡尔谱的方法}

用 Hesse 法 ${ }^{[1]}$ 或 Window 法 ${ }^{[2]}$ 拟合非晶合金谱的拟合质量依赖于亚谱六个峰的强度比， 峰宽和平均同质异能移位等参数值的合理性. 但实际上,准确估计这些参数值是困难的,为了 得到好的拟合质量需要反复试算. 此外, 这两种拟合方法没有考虑到谱线非对称性,也不能拟 合晶态组分谱与非晶组分谱混迭的复杂谱. 针对上述问题, 本文在上面对谱线非对称性分析 的基础之上,提出了一种改进的拟合方法.

1. 数学模型从最一般谱的拟合出发，设整个谱由晶态合金组分和超精细场呈连续分 布的非晶态组分的两种谱组成，其中晶态组分有 $M_{1}^{\prime \prime}$ 个亚谱，将非晶组分的谱划分为 $M_{1}^{\prime}$ 个 亚谱,共有 $M_{1}=M_{1}^{\prime}+M_{1}^{\prime \prime}$ 个亚谱. 设谱的全部待拟合的 $M$ 个参数构成参数向量 $q-\left(q_{1}\right.$, $\left.q_{2}, \cdots q_{M}\right)^{T}$. 将这 $M$ 个参数分为两大类: $\left(M_{1}+3\right)$ 维线性参数向量 $q_{1}$, 其中包括非晶组分㙕 的 $M_{1}^{\prime}$ 个亚谱强度 $q_{i}\left(i=1,2, \cdots M_{1}^{\prime}\right)$, 晶态组分谱的 $M_{1}^{\prime \prime}$ 个亚谱强度 $q_{i}\left(i=M_{1}^{\prime}+1, M_{1}^{\prime}+\right.$ $2, \cdots M_{1}$ ) 和三个抛物线型本底参数 $E, F, G$ (分别用 $q_{M_{1}+1}, q_{M_{1}+2}$ 和 $q_{M_{1}+3}$ 表示); $M_{2}$ 维 非线性参数向量 $\boldsymbol{q}_{11}-\left(q_{M_{1}+4}, q_{M_{1}+5}, \cdots q_{M}\right)^{T}$,其中包括非晶组分谱的参数 $a, b, c, d$, 各亚谱的 六个峰的归一化强度 $a_{K}$, 峰宽 $\Gamma_{i K}$ 以及晶态组分亚谱中待修正拟合的超精细参数 $H_{\mathrm{hf}} 、 \Delta E Q$ 和 $\delta$ 等.

设理论谱在第 $n$ 道上的计数为

$$
\mathrm{Y}_{t}(n)=E+F \cdot X_{n}+G \cdot X_{n}^{2}-\sum_{i=1}^{M_{1}} s_{i} \cdot \sum_{K=1}^{6} \frac{a_{K}}{1+4\left(X_{n}-P_{i K}\right)^{2} / \Gamma_{i K}^{2}},
$$

式中 $S_{i}$ 为第 $i$ 套亚谱的强度, $P_{i K}$ 为第 $i$ 套亚谱的第 $K$ 个峰的峰位, $X_{n}$ 为第 $n$ 道的速度. 改 
进的拟合方法取目标函数为

$$
\begin{aligned}
\chi_{m}^{2}- & \sum_{n=1}^{N}\left[Y_{c}(n)-Y_{t}(n)\right]^{2} / Y_{c}(n)+g \cdot \sum_{i=2}^{M_{1}^{\prime}-1}\left(\Delta q_{i-1}-2 \Delta q_{i}+\Delta q_{i+1}\right)^{2} \\
& +\lambda \cdot \sum_{i=1}^{M} \sum_{n=1}^{N}\left(\frac{\partial Y_{t}(n)}{\partial q_{i}} \cdot \Delta q_{i}\right)^{2} / Y_{c}(n),
\end{aligned}
$$

式中 $Y_{e}(n)$ 为实测谱在第 $n$ 道上的计数, $N$ 为谱的总的道数, $g$ 为光滑因子, $\lambda$ 为阻尼因子. 各参数的修正量 $\Delta q-\left(\Delta q_{1}, \Delta q_{11}\right)^{T}=\left(\Delta q_{1}, \Delta q_{2}, \cdots \Delta q_{M}\right)^{T}$ 由 $\chi_{m}^{2}$ 取极小值的条件确定, 经 线性化处理可得到矩阵形式的迭代方程:

$$
\left(\boldsymbol{A}^{[l]}+g \cdot \boldsymbol{D}+\lambda^{[l]} \cdot \boldsymbol{C}^{[l]}\right) \Delta \boldsymbol{q}^{[l]}=\boldsymbol{B}^{[l]},
$$

式中的光滑矩阵

$$
\boldsymbol{D}=\left(\begin{array}{cc}
\boldsymbol{D}_{1} & 0 \\
0 & 0
\end{array}\right), D_{11}=\left(\begin{array}{rrrrrrrr}
1 & -2 & 1 & & & & \\
-2 & 5 & -4 & 1 & & & \\
1 & -4 & 6 & -4 & 1 & & \\
& & \ddots & & & \ddots & \ddots & \\
& & & 1 & -4 & 6 & -4 & 1 \\
& & & 1 & -4 & 5 & -2 \\
& & & & 1 & -2 & 1
\end{array}\right),
$$

其他各矩其阵的矩阵元为

$$
\begin{aligned}
& A_{i j}^{[l]}=\sum_{n=1}^{N}\left[\frac{\partial Y_{t}(n)}{\partial q_{i}}\right]^{[l]} \cdot\left[\frac{\partial Y_{t}(n)}{\partial q_{i}}\right]^{[l]} / Y_{c}(n), \\
& C_{i j}^{[n]}= \begin{cases}0 & (i \neq j), \\
A_{i j}^{[l]} & (i-i),\end{cases} \\
& B_{i}^{[l]}=\sum_{n=1}^{N}\left[Y_{c}(n)-Y_{i}^{[l]}(n)\right] \cdot\left[\frac{\partial Y_{t}(n)}{\partial q_{i}}\right]^{[l]} / Y_{c}(n),
\end{aligned}
$$

式中的 $i, j-1,2, \cdots M$.

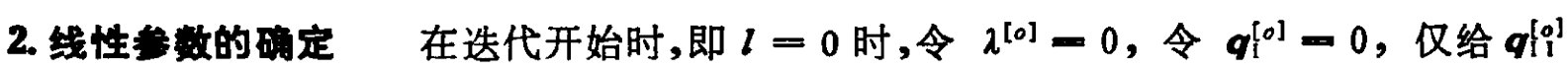
践予适当的初值. 在上述条件下,理论谱对所有非线性参数的导数均为零. 因此,方程 (5) 简 化为

$$
\left(\begin{array}{cc}
A[1] & 0 \\
0 & 0
\end{array}\right)\left(\begin{array}{c}
\Delta q_{1}^{[o]} \\
\Delta q_{11}^{[o]}
\end{array}\right)+g\left(\begin{array}{cc}
D_{11} & 0 \\
0 & 0
\end{array}\right)\left(\begin{array}{c}
\Delta q_{1}^{[o]} \\
\Delta q_{11}^{[o]}
\end{array}\right)-\left(\begin{array}{c}
B_{1}^{[o]} \\
0
\end{array}\right) \text {. }
$$

由此可得第 0 次迭代时参数修正量的合理值为

$$
\left\{\begin{array}{l}
\Delta \boldsymbol{q}_{1}^{[0]}=\left(\boldsymbol{A}_{1 !}^{[o]}+g \boldsymbol{D}_{11}^{[0]}\right)^{-1} \boldsymbol{B}_{1}^{[o]}, \\
\Delta \boldsymbol{q}[1]=0
\end{array}\right.
$$

各参数值

$$
\left\{\begin{array}{l}
\boldsymbol{q}^{[1]}=\boldsymbol{q}_{1}^{[0]}+\Delta \boldsymbol{q}^{[0]}=\left(\boldsymbol{A}_{11}^{[0]}+g \cdot \boldsymbol{D}_{11}\right)^{-1} \boldsymbol{B}_{1}^{[o]}, \\
\boldsymbol{q}\left[\left.\right|^{[1]}=\boldsymbol{q}_{11}^{[0]}+\Delta \boldsymbol{q}_{11}^{[0]}=\boldsymbol{q}_{11}^{[0]} .\right.
\end{array}\right.
$$

将式 (12) 与 Hesse 法的结果 ${ }^{[1]}$ 对比可知,对于解非晶合金谱来说, 除了权重的考虑和基线参 数 $E 、 F 、 G$ 可直接由式(12)解出这两个特点外,两种解谱方法在本质上完全相同. 因此,在第 
0 次迭代时上述方法是一种改进的 Hesse 法.

3. 数的迭代传正 $\quad l=1$ 时, 开始对全部参数进行迭代修正. 为了改善迭代修正的 收玫性,可以赋给阻尼因子 $\lambda^{[l]}$ 适当的正值. 将 $\boldsymbol{q}^{[1]}$ 做为新的参数初值, 则可由方程 (5) 求 得全部参数的修正值 $\Delta q^{[1]}$. 因此, 新的参数值 $q^{[2]}-q^{[1]}+\Delta q^{[1]}$. 重复这个过程, $q^{[l+1]}-$ $\boldsymbol{q}^{[l]}+\Delta \boldsymbol{q}^{[l]}$, 直到拟合谱的 $\chi^{2}$ 值满意为止. 因此, 在 $l \neq 0$, 上述迭代修正方法实质上是阻 尼最小二乘法的找谱方法.

4. 改进的拟合方法的讨论 由上述分析可知，本方法实质上是改进的 Hesse 法与一般 的非线性阻尼最小二乘法的有机结合. 本方法有以下几个特点:

1）不需要输人线性参数的初估值，因而拟合复杂的重迭严重的谱时使用比较方便.

2）对于解非昆合金谱,具有修正参数的功能, 从而可以提高谱的拟合质量.

3）适用范围广，既能解非晶合金谱，又能解晶相组分和非晶组分迭加的混合谱或纯晶态 谱, 是一个通用的解谱方法.

4) 对于由已知物相和未知物相谱构成的混合谱, 具有剥谱和拟合的混合运算功能 ${ }^{[7]}$.

\section{三、改进的拟合方法应用举例}

用上述方法拟合 $\mathrm{Fe}_{78} \mathrm{Si}_{10} \mathrm{~B}_{12}$ 急冷非晶合金在回火前后的室温稞斯堡尔谱均得到比较满意 的拟谱质量, 拟合的谱参数列于表 2. 拟合结果表明, 谱线非对称性参数 $a$ 和 $b$ 随回火温度上 升逐渐变小，反映了谱线非对称性不仅与合金的成分有关，而且与热处理状态有关. 对 CoFe-B-Si 非晶合金谱的拟合也得到良好的结果 ${ }^{[8]}$.

表 $2 \mathrm{Fe}_{78} \mathrm{Si}_{10} \mathrm{~B}_{12}$ 急冷非晶合金谱的参数

\begin{tabular}{c|c|c|c|c|c|c|c|c}
\hline 样 品 & $a_{2} / a_{3}$ & $a \times 10^{3}$ & $j b \times 10^{3}$ & $c(\mathrm{~mm} / \mathrm{s})$ & $d(\mathrm{~mm} / \mathrm{s})$ & $\begin{array}{c}\bar{H}_{\mathrm{bf}} \\
(\mathrm{KOc})\end{array}$ & $\bar{\delta}(\mathrm{mm} / \mathrm{s})$ & $\begin{array}{c}\Delta \bar{E} Q \\
(\mathrm{~mm} / \mathrm{s})\end{array}$ \\
\hline 急冷制备态 & 2.47 & -1.06 & 0.48 & 0.26 & -0.01 & 251.5 & 0.11 & -0.01 \\
\hline $385^{\circ} \mathrm{C}$ 回火 & 2.44 & -1.16 & 0.40 & 0.30 & 0.02 & 255.5 & 0.12 & 0.01 \\
\hline $440^{\circ} \mathrm{C}$ 回火 & 2.02 & -1.30 & 0.26 & 0.32 & 0.05 & 257.8 & 0.12 & -0.01 \\
\hline
\end{tabular}

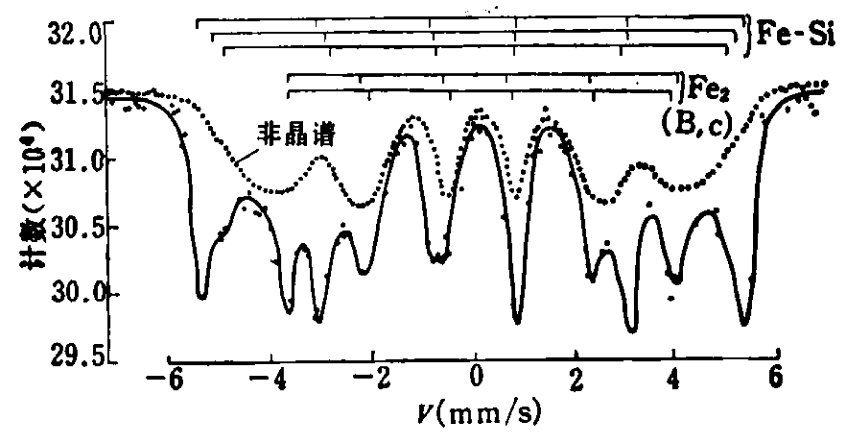

图 2 部分晶化的 $\mathrm{Fe}_{21} \mathrm{~B}_{13}, \mathrm{Si}_{3 .}, \mathrm{C}_{2}$ 非晶合金徣

对部分晶化的 $\mathrm{Fe}_{81} \mathrm{~B}_{13.5} \mathrm{Si}_{3.5} \mathrm{C}_{2}$ 非晶合金 $\left(450^{\circ} \mathrm{C} \times 1 \mathrm{~h}\right.$ 回火) 室温穆斯堡尔谱 (图 2) 的拟 合结果表明,该样品的晶态组分为 $\mathrm{Fe}-4$ at \% Si 和 $\mathrm{Fe}_{2}(\mathrm{~B}, \mathrm{C})$, 非晶组分占 $50 \%$ 左右. 图 3 是 对完全晶化的 $\mathrm{Fe}_{80} \mathrm{P}_{17.6} \mathrm{Si}_{1.6} \mathrm{C}_{0.8}$ 非晶合金室温穆斯堡谱的拟合结果.

综上所述,在考虑到三个超精细参数 $H_{\mathrm{hf}} 、 \Delta E Q$ 和 $\delta$ 之间共存的相关性的基础上, 用本 


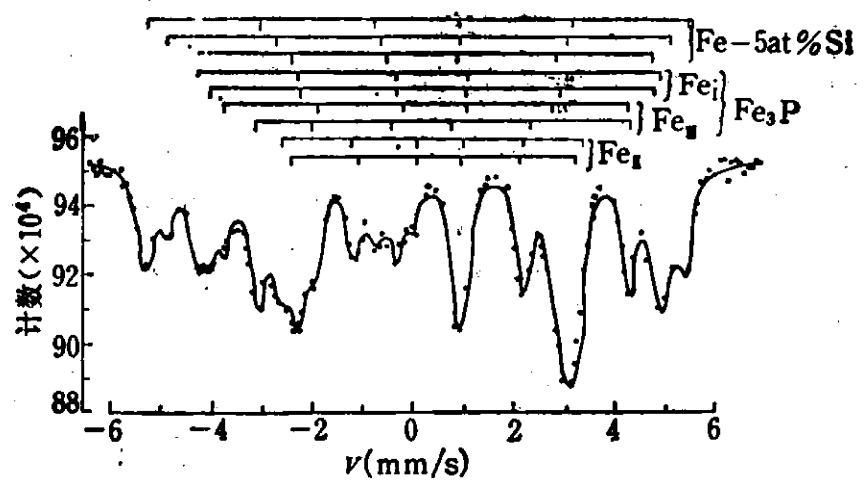

图 3 宗全晶化的 $\mathrm{Fe}_{20} \boldsymbol{P}_{21.0} \mathrm{Si}_{\mathbf{2} .6} \mathrm{C}_{0.8}$ 非晶合金谱

文提出的拟合方法可以有效地提高非晶合金谱的拟合质量. 改进的拟合方法具有拟谱功能 多,适用范围广和使用方便的优点.

\section{$\Rightarrow$ 文}

[ 1 ] Hesse, J. and Rubartsch, A., J. Phys.x E7(1974), 526.

[ 2 ] Window, B., J. Phys., E4(1971), 401.

[ 3 ] Chien, C. L. and Chen, H. S., J. de Phys. Colloq, 1979, C2-118.

[ 4 ] Logan, J. and Sun, E., J. Non-cryst. Solids, 20(1976), 285.

[ 5 ] Price, D. C. et al., J. de Phys. Colloq., 1980, C1-263.

[6] Caer, G. Le and Dubois, J. M., Phys. Stat. Sol. (A), 64(1981), 275.

[7] 本士等,科学通报, 27(1982),6: 335.

[8] 本国标、本士等,科学通报, 28(1983)，20: 1227. 